\title{
On a Stable and Consistent Finite Difference Scheme for a Time-Dependent Schrodinger Wave Equation in a Finitely Low Potential Well
}

\author{
${ }^{*}$ O.J. Adeleke, ${ }^{2}$ M.O. Oke and ${ }^{3}$ A.O. Adewumi \\ 1Department of Computer and Information Science/Mathematics Covenat University, Ota. Nigeria. \\ 2Department of Mathematical Sciences, Ekiti State University, Ado-Ekiti. \\ ${ }^{3}$ School of Maths., Statistics \& Computer Science, University of KwaZulu-Natal, Westville Campus, Durban, South Africa \\ [Correspondence author Email: wale.adeleke@covenantuniversity.edu.ng]
}

\begin{abstract}
In this paper, a stable and consistent criterion to an explicit finite difference scheme for a timedependent Schrodinger wave equation (TDSWE) was presented. This paper is a departure from the wellestablished time independent Schrodinger Wave Equation (SWE). To develop the stability criterion for the scheme, the Fourier series method of von Newmann was adopted, while in establishing the consistency property, the concise definition of the consistent scheme was applied. This research is carried out for a particular case of a finitely low potential well.
\end{abstract}

Keywords: Time-Dependent Schrodinger Wave Equation, Stability, Consistency, Finite Potential Well, Finite Difference.

\section{INTRODUCTION}

Two forms of the Schrodinger wave equation (SWE) exist. In the first, the time factor is explicitly expressed, for which reason it is widely referred to as the timedependent SWE. In the second, known as the timeindependent SWE, the time factor is removed. In practice though these two equations are separate, the latter, which is the time-independent SWE can be derived from the former. The only exception to this order is if the potential is time dependent.

The Schrodinger equation, which was developed by Erwin Schrodinger, is very central to the study of quantum mechanics as it defines the permissibility of a stationary state of a quantum mechanics. Accordingly, it tells how the quantum state of a physical system changes with time (Schrodinger Equation (Physics), Encyclopedia Britannica).

\section{Time-Dependent SWE}

According to Shanker (1994), the form of Schrodinger equation depends on the physical situation. The most general form is the time-dependent Schrodinger equation, which gives a description of a system evolving with time.

This time-dependent SWE is given as

$-\frac{\hbar^{2}}{2 m} \nabla^{2} \psi(x)+v(x) \psi=i \hbar \frac{\partial \psi}{\partial t}$ where $\hbar=\frac{\hbar}{2 \pi}, h$ being the Planck's constant, $m$ is

the mass of particle, $\psi(x, t)$, is the wave function, $x$ is

the position of any particular particle in time $t, v(x)$ is

the time-dependent potential, and $t$ is the time.

When this equation is set up for analysis, it forms the bedrock for wave mechanics, a branch of quantum mechanics. This equation, when solved, is capable of generating solutions that depict a wave propagating through space. This explains the rationale behind being termed a wave equation even though it does not represent properly the more familiar classical wave equation.

Koch (2004) developed a numerical scheme that is workable with the TDSWE in an Ultrafast Laser e. The scheme so developed by Koch approximates the original wave equation on a linear manifold.

With basis on a discretizing space and time on a grid, Kosloff and Kosloff (1983) developed a new method through the Fourier method to produce spatial derivatives and the second-order differencing for time derivatives. Petridis et al. (2010), by means of superposition of time-independent solution with the approximated spectral functions, developed an exact solution to the TDSWE which satisfied both the boundary and square-integrability conditions. In a thesis 
by Persson (2012), different numerical methods for solving the time-dependent SWE were described. The spectral and pseudospectral representations of the equation were described and subsequently subjected to the different numerical techniques with some practical examples to serve for illustrations. A novel numerical method that allows the computation of the exact solutions of a stationary Schrodinger equation was proposed by Rieth et al. (2002). It was affirmed by the the authors that well-known reference system will be needed for the practical application of the method. The said method was proposed for the determination of the eigenfunctions and eigenvalues of a particle in a quantum-mechanized system.

We follow a similar numerical approach in this paper. For the TDSWE, we develop a criterion for the stability of a finite difference scheme to the TDSWE. The consistency of the scheme is also verified.

\section{Stability and Consistency Criteria for a Finite Difference Scheme}

To investigate the stability property of the finite difference scheme to be shortly developed, the Fourier series method has been adopted. This method developed by von Neumann, and first discussed by O'Brian et al (1951), "expressed an initial line of errors in terms of a finite Fourier series, and considers the growth of a function that reduces to this series for $t=0$ by a separation of variable method commonly used for solving analytically a partial differential equation (Smith, 1978).

The procedure for doing this is as follows.

Suppose we denote the error at the pivotal point (Smith, 2009) by

$$
\begin{gathered}
E(p h)=E_{p,} p=0(1) N \\
\equiv E_{p_{\ell} O}
\end{gathered}
$$

Then the $(N+1)$ equations

$E_{p}=\sum_{n=0}^{N} A_{n} e^{i \beta_{n} p h}, p=0(1) N$

(where $A_{n}$ is constant and $\beta_{n}=\frac{n \pi}{N h}, N h=1$ ) are sufficient to determine the unknown $A_{k}, k=0(1) n$, uniquely. For a linear finite difference

scheme ours with separate additive solutions, we need only consider the proportion of the error due to a single term, such as $e^{i \beta p h}$. $A_{n}$ being constant may be neglected.

For an increasing $t$, investigating this error, we need only to find a solution which reduces to $e^{i \beta p h}$ when $t=q k=0$.

If we assume

$E_{p, q}=e^{i \beta x} e^{\alpha t}=e^{i \beta p h} e^{\alpha q k}=e^{i \beta p h} \xi^{q}$

Where $\xi=e^{\alpha k}$ with $\alpha_{x}$ in general, a complex constant.

When $q=0, E_{p, q}=e^{i \beta p h}$.

The only way to keep the error stable as $t$ increases is to make a provision such that

$$
|\xi| \leq 1
$$

Equation (5) is known as the stability criterion. It is both necessary and sufficient for two time-level difference scheme but not always for three time-level schemes (Smith, 2009).

For a more concise approach, suppose we take $e=U-u$, where $e$ is the discretization error, $U$

is the exact solution of the partial differential equation (pde) and $u$, the exact solution of the finite difference

scheme. Then, if $u_{m, n}$ is bounded as $n$ increases, the difference equation is said to be stable. In a nutshell, if 
$e_{m, n}=U_{m, n}-u_{m, n}$ remains bounded as $n \rightarrow \infty$, then the difference equation to the pde is stable.

In a similar fashion, suppose $F_{m, n}(u)=0$ is a difference equation at $(m, n)$ th mesh point, then a finite difference is said to be consistent with the pde if $F_{m, n}(u) \rightarrow 0$ as $\delta x, \delta t \rightarrow 0$.

\section{RESULTS AND DISCUSSION}

In what follows in this paper, we present the results for both stability and consistency of the explicit finite difference equation for TDSWE. We begin by giving the difference equation.

The time dependent Schrodinger equation wave equation is

$-\frac{\hbar^{2}}{2 m} \nabla^{2} \psi(x)+v(x) \psi=i \hbar \frac{\partial \psi}{\partial t}$

We can write this as

$$
\begin{aligned}
& -\frac{\hbar^{2}}{2 m} \frac{\partial^{2} \mathrm{u}}{\partial x^{2}}+v(x) \mathrm{u}=i \hbar \frac{\partial \mathrm{u}}{\partial t}, 0<x<a, t>0 \\
& \operatorname{Let} \frac{\hbar^{2}}{2 m}=-c^{2} \text { and } i h=d \\
& \frac{\partial \mathrm{u}}{\partial t}=\frac{u_{m, n+1}-u_{m, n}}{k} \text { and } \frac{\partial^{2} \mathrm{u}}{\partial x^{2}}=\frac{u_{m+1, n}-2 u_{m, n+u_{m-1, n}}}{k} \\
& u=u_{m, n}
\end{aligned}
$$

For a simple case where $v$ is unity i.e $v(x)=1$

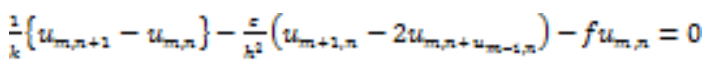

Where

$$
e=\frac{c^{2}}{d} \text { and } f=\frac{1}{d}
$$

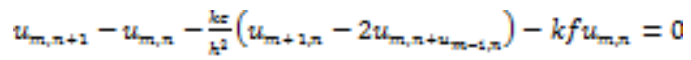

$$
\begin{aligned}
& u_{m_{m n+1}}-u_{m_{n} n}-r e u_{m_{m} n+1}-2 r e u_{m_{m} n}+w_{m-1, n}-k f u_{m, n}=0 \\
& u_{m_{m n+1}}+(2 r e-k f-1) u_{m_{m}}-r e\left(u_{m, n+1}-u_{m-1, n}\right)=0 \\
& u_{m, n+1}=r e\left(u_{m+1, n}-u_{m-1, n]+}(1+k f-2 r e) u_{m, n}\right.
\end{aligned}
$$

We thus come to the difference scheme given by

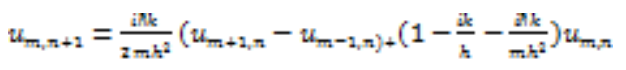

This equation is the finite difference scheme of the time-dependent Schrodinger wave equation in a finite potential well, where $v(x)=1$.

\section{Stability}

In developing a stability criterion for (13), we employ both Courant, Friedrichs andLewy Criteria [CFL criteria] (Courant et al., 1928) and the Von Neumann method (O'Brian et al., 1951). From the difference scheme for the classical wave equation, the former found that if $c \frac{\partial t}{\partial x}=c \frac{k}{h}>1$, then the scheme is unstable. The CFL

criteria has its origin in the fact that if $c \Delta t>\Delta x$, then

the rate at which signals in the numerical scheme travel will be faster than their real world counterparts and this unrealistic expectation leads to instability.

We proceed from the Von Neumann-Fourier method Let

$$
u_{m, n}=e^{-i m \theta} e^{i n \lambda}
$$

where $\theta$ is an arbitrary real number and $\lambda$ is a complex number yet to be determined. This choice is motivated by the fact that the initial condition $u_{m, 0}$ can be represented by a Fourier series where a typical term behaves as $e^{-i m \theta}$. 
By substituting (14) into (13) we obtain,

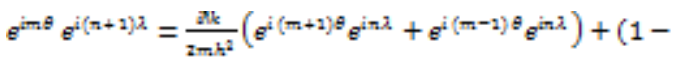

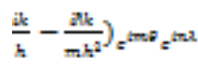

$e^{i \lambda}=\frac{i \hbar k}{2 m h^{2}} \sin ^{2}\left(\frac{\theta}{2}\right)+\left(1-\frac{i k}{h}-\frac{i \hbar k}{m h^{2}}\right)$

The quantity $e^{i \lambda}$ will grow exponentially unless

$-1 \leq e^{i \lambda}=\frac{i n k}{2 m h^{2}} \sin ^{2}\left(\frac{\theta}{2}\right)+\left(1-\frac{i k}{h}-\frac{i \pi k}{m h^{2}}\right)<1$

i.e

$-1 \leq 1-\frac{i k}{\hbar h}-\frac{i \hbar k}{m h^{2}}\left(1-\frac{1}{2} \sin ^{2}\left(\frac{\theta}{2}\right)\right)<1$

$0 \leq-1-\frac{\emptyset}{m h^{2}}\left(1-\frac{1}{2} \sin ^{2}\left(\frac{\theta}{2}\right)\right)<\frac{2}{\mathbb{i k}}$

$1 \leq-\frac{\hbar}{m h^{2}}\left(1-\frac{1}{2} \sin ^{2}\left(\frac{\theta}{2}\right)\right)<\frac{2}{i k}+1$

$\left\{\frac{m h^{2}}{\sharp} \leq-\left(1-\frac{1}{2} \sin ^{2}\left(\frac{\theta}{2}\right)\right)<\frac{m h^{2}}{\varpi}\left(1+\frac{2}{i k}\right)\right\}$

$1 \leq \frac{\hbar}{2 m h^{2}} \sin ^{2}\left(\frac{\theta}{2}\right)-\frac{\hbar}{m h^{2}}<1+\frac{2}{i k}$

Taking the right hand side of (22), i.e.,

$1-\frac{i k}{h}-\frac{i \hbar k k}{m h^{2}}\left(1-\frac{1}{2} \sin ^{2}\left(\frac{\theta}{2}\right)\right)<1$

$\frac{\hbar^{2} k}{m h^{2}}>\frac{k}{\frac{\sin ^{2}}{2}\left(\frac{\theta}{2}\right)-1}$

$$
\frac{\hbar^{2} k}{2 m h^{2}}\left(\sin ^{2}\left(\frac{\theta}{2}\right)-2\right)>k
$$

$$
\frac{\hbar^{2} k}{2 m h^{2}}>\frac{k}{\sin ^{2}\left(\frac{\theta}{n}\right)-2}
$$

For the left hand side inequality of (22) we have

$\left.1 \leq \frac{\hbar}{2 m h^{2}} \sin ^{2}\left(\frac{\theta}{2}\right)\right)+1-\frac{i k}{\hbar}-\frac{i k h}{m h^{2}}$

$\frac{k}{\left.\left(\sin ^{2}\left(\frac{\theta}{2}\right)\right)-2\right)}<\frac{\hbar^{2} k}{2 m h^{2}} \leq \frac{k+2 i \hbar}{\left.\left(\sin ^{2}\left(\frac{\theta}{2}\right)\right)-2\right]}$

That is,

$\frac{k}{\left(\sin ^{2}\left(\frac{g}{2}\right) b-2\right)}<\frac{n^{2} k}{2 m h^{2}} \leq \frac{k}{\left(\sin ^{2}\left(\frac{(g)}{2}\right) b-2\right)}+\frac{2 i n}{\left(\sin ^{2}\left(\frac{(g)}{2}\right) b-2\right]}$

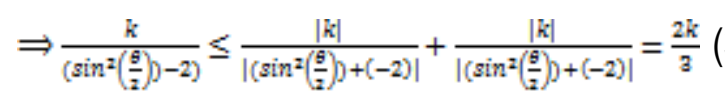

Combining inequalities (26) and (30), we get

$$
\frac{k}{\sin ^{2}\left(\frac{\theta}{2}\right)-2}<\frac{\hbar^{2} k}{2 m h^{2}} \leq \frac{2 k}{3}
$$

The result equation (31) gives our proposed stability criterion for an explicit finite difference scheme of the TDSWE.

\section{Consistency}

We know that

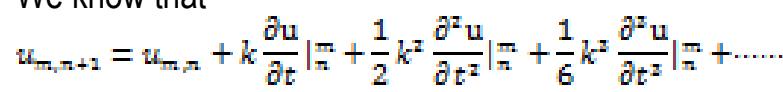

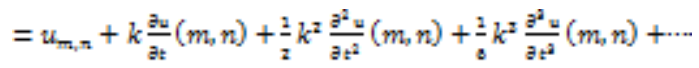

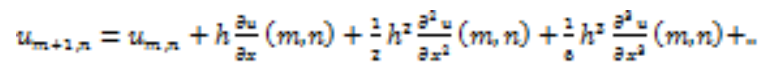

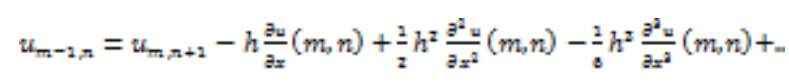


By substituting (32) to (34) into (13), we obtain

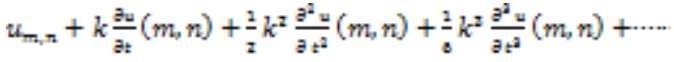

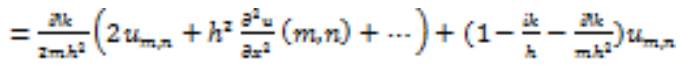

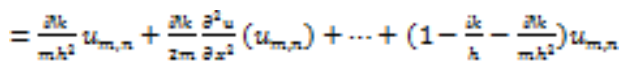

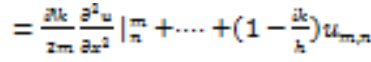

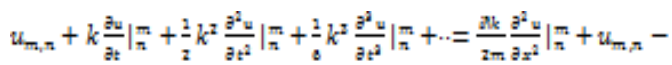

$$
\begin{aligned}
& \frac{\sqrt{k}}{4} u_{m, n}
\end{aligned}
$$

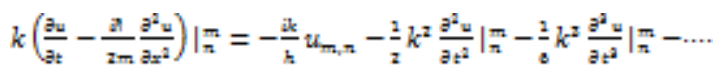

The term on the LHS vanishes because $u(x, t)$ satisfies the wave equation. Likewise, as $k=\Delta t \rightarrow 0$, the terms on the RHS tend to zero(0)

and so (13) is a consistent finite difference approximation to the Schrodinger wave equation.

Table 1: Computational Results with $h=0.1$ and $t=\frac{1}{400}$

\section{Numerical Results}

To establish the validity of the result in (31) above, we take the values of the step sizes as

$h=0.1$ and $k=1 / 400$ with the boundary values as

$0 \leq x \leq 0.5$ and $t>0$. The value of the mesh ratio

is given by the inequality $r \leq 1.054 \times 10^{25}$. This

value was computed based on the values of $\hbar=1.054772727 \times 10^{-34} \quad$ and

$m=3.52 \times 10^{-41}$. This result satisfies the stability

criterion in (31).

In the following table, the numerical results obtained on a Maple 14 software are reported.

\section{CONCLUSION}

In the course of this paper, we have been able to develop criteria for both the stability and consistency for the difference equation of a time-dependent Schrodinger wave equation. The consequential effect of these results is that the scheme is convergent as it approaches a continuous solution as $\Delta x, \Delta t \rightarrow 0$. In

the future, we hope to extend the Schrodinger wave equation for cases of two and three dimensions.

\begin{tabular}{|c|c|c|c|c|c|c|}
\hline$t$ & $x=0$ & $x=0.1$ & $x=0.2$ & $x=0.3$ & $x=0.4$ & $x=0.5$ \\
\hline 0 & $1.0+0 . i$ & $1.0-5.0 \times 10^{-11} i$ & $1.0+0 . i$ & $1.0+1.0 \times 10^{-10} i$ & $1.0-1.1 \times 10^{-9} i$ & $1.0+0 . i$ \\
\hline$\frac{1}{400}$ & $0.9-0.4 i$ & $0.9-0.4 i$ & $0.9-0.4 i$ & $0.9-0.4 i$ & $0.9-0.4 i$ & $0.9-0.4 i$ \\
\hline$\frac{2}{400}$ & $0.7-0.7 i$ & $0.7-0.7 i$ & $0.7-0.7 i$ & $0.7-0.7 i$ & $0.7-0.7 i$ & $0.8-0.7 i$ \\
\hline$\frac{3}{400}$ & $0.4-0.9 i$ & $0.4-0.9 i$ & $0.4-0.9 i$ & $0.4-0.9 i$ & $0.4-0.9 i$ & $0.4-0.8 i$ \\
\hline$\frac{4}{400}$ & $0.1-1.0 i$ & $0.1-1.0 i$ & $0.1-1.0 i$ & $0.1-1.0 i$ & $0.1-1.0 i$ & $0.1-1.0 i$ \\
\hline$\frac{5}{400}$ & $-0.3-1.0 i$ & $-0.3-1.0 i$ & $-0.3-1.0 i$ & $-0.3-1.0 i$ & $-0.3-1.0 i$ & $-0.3-1.0 i$ \\
\hline
\end{tabular}




\section{REFERENCES}

Courant, R., Friedrichs, K. and Lewy, H. (1967). On the Partial Difference Equations of Mathematical Physics. IBM Journal of Research and Development, 11: 215-234.

Koch, O. (2004). Numerical solution of the timedependent Schrodinger equation in ultrafast laser dynamics. AURORA Special Research Program. Austria.

Kosloff, D. and Kosloff, R. (1983). A Fourier method solution for the time-dependent Schrodinger equation as a tool in molecular dynamics. Journal of Computational Physics, 52: 35-53.

O'Brian, C.G., Hyman, M.A. and Kaplan, S. (1951). A study of the numerical solution of partial differential equations. Journal of Mathematical Physics, 21: 232-237.

Persson, A. (2012). Numerical methods for solving the time-dependent Schrodinger equation. Bachelor's thesis, Division of Mathematical Physics, Luud University.
Petridis, A. N., Staunton, L. P., Vermedahl, J. and Luban, M. (2010). Exact analytical and numerical solutions to the time-dependent Schrodinger wave equation for a one-dimensional potential exhibiting non-exponential decay at all times. Journal of Modern Physics, 1: 124-136.

Rieth, M., Schommers, W. and Baskoutas, S. (2002). Novel numerical method for the solution of Schrodinger's equation: I. A particle in an interaction potential of general shape. International Journal of Modern Physics B, 16: 4081-4092.

Schrodinger equation. 2014. Encyclopædia Britannica Online. Retrieved 18 January, 2014, from http://www.britannica.com/EBchecked/topic/5282 98/Schrodinger-equation

Shankar, R. (1994). Principles of quantum mechanics, $2^{\text {nd }}$ ed., Kluwer Academic/Plenum Publisher, $p$. 143.

Smith, G.D. (1978). Numerical computation of partial differential equations. Oxford University Press. $2^{\text {nd }}$ edition. 